\title{
Periodic breathing in healthy humans at exercise in hypoxia.
}

\section{Running head: Intrinsic periodic breathing in humans}

Eric Hermand ${ }^{1}$, Aurélien Pichon ${ }^{1}$, François J Lhuissier ${ }^{1,2}$, Jean-Paul Richalet ${ }^{1,2}$

${ }^{1}$ Université Paris 13, Sorbonne Paris Cité, Laboratoire “Hypoxie et poumon”, EA2363, Bobigny, France

${ }^{2}$ Assistance Publique-Hôpitaux de Paris, Hôpital Avicenne, Service de Physiologie, explorations fonctionnelles et médecine du sport, 93009 Bobigny, France

\section{Corresponding author}

Pr. Jean-Paul Richalet

Laboratoire "Hypoxie et poumon", EA2363

74 rue Marcel Cachin

93017 Bobigny Cedex, FRANCE

Tel: 33148387758 / Fax: 33148388924

Email: richalet@univ-paris13.fr

Running head: intrinsic periodic breathing

Word count for manuscript: 3430 


\begin{abstract}
Periodic breathing is frequent in heart failure or ventilatory disorders during sleep, and common during sleep at high altitude, but has been rarely studied in wakefulness and during exercise. A retrospective analysis of ventilation from hypoxia exercise tests was realized in 82 healthy subjects, separated in two groups with either high or low ventilatory response to hypoxia at exercise (HVRe). A FFT spectral analysis of the breath-by-breath ventilation $\left({ }_{\varsigma} \mathrm{E}\right)$ signal, $\mathrm{O}_{2}$ saturation and end-tidal $\mathrm{PCO}_{2}$ evidenced a periodic pattern with a period of 11.1 to 12.0 seconds. Peak power of $\varsigma_{\mathrm{E}}$ spectrum was higher in the high HVRe group $(P<0.001)$. A prospective study (25 subjects) was performed to evaluate the influence of cardiorespiratory factors on the amplitude and period of oscillations in various conditions of exercise (20 to $40 \%$ maximal aerobic power) and hypoxia (0 to $4,000 \mathrm{~m}$ altitude). $\varsigma \mathrm{E}$ period was shorter at exercise (vs rest, $P<0.001$ ) and hypoxia (vs normoxia, $P<0.001$ ) and inversely related with cardiac output and $\varsigma \mathrm{E}$ $(\mathrm{P}<0.001)$. $\varsigma \mathrm{E}$ peak power was higher at exercise $(P<0.001)$ and hypoxia $(P<0.001)$ and was positively related with cardiac output and ${ }_{\varsigma} \mathrm{E}(P<0.001)$. $\varsigma_{\mathrm{E}} \mathrm{E}$ peak power in hypoxia was positively related with the ventilatory response to $\mathrm{CO}_{2}$ (HCVR). This novel observation suggests that healthy subjects demonstrate a spontaneous periodic breathing, not clearly observable at rest and in normoxia, but triggered by hypoxic exercise. The periodic pattern is enhanced in subjects with high HVRe and high HCVR, suggesting that oxygen and $\mathrm{CO}_{2}$ play a synergistic role in the modulation of these oscillations.
\end{abstract}

Clinical Trial reg. $\mathrm{n}^{\circ}$ : NCT02201875

\title{
Word count: 250
}

\section{Key words:}

Hypoxia

Control of ventilation

Periodic breathing

Hypoxic ventilatory response

Hypercapnic ventilatory response

Exercise 


\section{Introduction}

The control of ventilation involves three main components: the respiratory centers, responsible for the genesis of the respiratory rhythm, the effector system (the ventilatory muscles) and the receptors (mainly the chemoreceptors) that inform the centers of the status of the whole system. Central chemoreceptors are sensitive to the level of arterial $\mathrm{PCO}_{2}$ with gain adjustments from peripheral chemoreceptors $(5,25)$, although these interactions have been debated in humans (16), while the response to hypoxia is mainly due to peripheral chemoreceptors (carotid bodies) $(23,26)$. All these components are involved in a closed loop system of control that produces, in most usual circumstances, a relatively stable level of ventilation adapted to the needs of the organism. However, in some cases, the system shows a marked degree of instability with periodic breathing or interruption of ventilation. Sleep apneas or hypopneas might be of obstructive or central origin and are accompanied by oscillations in the level of end tidal $\mathrm{PCO}_{2}$ and arterial $\mathrm{O}_{2}$ saturation $(2,46)$. Indeed, patients with cardiac failure may show a periodic breathing or Cheyne-Stokes pattern of ventilation at rest $(7,35,37,41)$ and during exercise $(13,14)$. Normal subjects may also show a periodic breathing pattern during sleep at high altitude $(1,3,24,29,30)$. The factors affecting breathing instability have been extensively discussed $(11,22)$. However, studies made in awake subjects at high altitude are very scarce $(8,10,17,31,42)$, and only one observation of periodic breathing during exercise at high altitude, in field conditions, has been reported (20).

The instability of the ventilation control system in patients is related to three main factors: the low reserve of $\mathrm{O}_{2}$ and $\mathrm{CO}_{2}$ in the lungs and tissues, the delay of signal transmission between the lungs and the central/peripheral chemoreceptors, and a high gain of the chemoreceptor response to $\mathrm{O}_{2}$ or $\mathrm{CO}_{2}$ or of the central response to input signals $(11,34)$. A physiological or environmental stress can reveal the unstable nature of the ventilatory control system. This can be initiated by a mechanical (obstruction of airways), chemical (rapid variation of $\mathrm{O}_{2}$ or $\mathrm{CO}_{2}$ pressures) or central (sigh) constraint (12).

When ventilation was recorded breath-by-breath, a periodic breathing was observed in healthy subjects coming to our outpatient mountain medicine consultation at Avicenne hospital in Bobigny (France). The ventilation signal showed a periodic breathing pattern, which increased when the subject started to exercise in hypoxic conditions and was subsequently maintained during normoxic exercise. During this consultation, subjects perform a hypoxia exercise test, which allows a good prediction of their risk factors for severe high altitude illnesses $(9,39)$. 
Therefore, our objective was to confirm this observation of periodic breathing in healthy subjects in a retrospective study led in 82 subjects who performed this hypoxia exercise test. We hypothesized that subjects with a high ventilatory response to hypoxia might show a more pronounced periodic pattern of ventilation, due to a higher gain of the chemoreceptor feedback loop. Then, in a prospective study conducted in 25 subjects, we determined the influence of cardiorespiratory parameters (ventilation, cardiac output) on the observed periodic pattern, at different levels of exercise intensity and hypoxic stress. We also conducted a $\mathrm{CO}_{2}$ rebreathing test on each subject to assess the potential role of central chemoreceptors $(15,38)$ on the genesis of ventilation oscillations.

If the hypothesis were confirmed, periodic breathing would not be considered as a rare phenomenon observed in some patients or in normal subjects in hypoxic conditions, but as a common characteristic of the breathing pattern in humans, exacerbated in some pathological or physiological conditions. The observation of this phenomenon in high-risk patients may help the early detection of patients susceptible to develop apneic syndromes.

\section{Subjects and methods}

\section{Retrospective study}

Subjects.

Among the population coming to the outpatient consultation of mountain medicine at Avicenne hospital in 2012, 82 subjects (38 females and 44 males) were retrospectively randomly selected and separated in two groups of 41 high and 41 low responders to hypoxia according to the median value of the hypoxic ventilatory response to hypoxia at exercise (HVRe $>$ or $<0.84$ L. $\mathrm{min}^{-1} \cdot \mathrm{kg}^{-1}$ ) derived from the hypoxic exercise test (inspired fraction of $\mathrm{O}_{2}: 0.115$, exercise intensity of $30 \%$ of maximal aerobic power), as previously described $(9,27,28,38)$. Characteristics of the two groups are shown in table 1.

\section{Procedure.}

The hypoxic exercise test consists in 4 successive phases of 3 to 4 minutes each with the following sequence: rest in normoxia $(\mathrm{RN})$, rest in hypoxia $(\mathrm{RH})$, exercise in hypoxia $(\mathrm{EH})$ and exercise in normoxia (EN) (Figure 1). Minute ventilation $\left(\varsigma \mathrm{E}, \mathrm{L} \cdot \mathrm{min}^{-1}\right)$ was measured through a metabograph (Vmax Encore, SensorMedics, Yorba Linda, CA). Tidal volume (VT), total respiratory cycle time (Ttot) and inspiratory time (Ti) were derived from the ventilation signal. Pulse $\mathrm{O}_{2}$ saturation $\left(\mathrm{SpO}_{2}, \%\right)$ was measured by transcutaneous oximetry (Nellcor N-595, Nellcor, Pleasanton, $\mathrm{CA})$ on a pre-warmed ear lobe. End tidal $\mathrm{PCO}_{2}\left(\mathrm{PETCO}_{2}\right)$ was measured by 
infrared thermopile (Vmax Encore, SensorMedics, Yorba Linda, CA). As previously defined, ventilatory response to hypoxia at exercise was measured as the ratio of increase in $\varsigma \mathrm{E}$ over the decrease in $\mathrm{SpO} 2$ between normoxic and hypoxic conditions at exercise $(6,9,27,28,39)$. During the whole test, $\varsigma \mathrm{E}, \mathrm{SpO}_{2}$ and $\mathrm{PETCO} 2$ were recorded breath-by-breath (Figure 1). Data were transferred to a computer for further variability analysis. A Fast Fourier Transform (FFT) was then applied to the ventilation signal in sequences of 128 seconds of a steady state interval in each phase of the test. This method allowed us to detect the presence of peaks in the frequency domain of the ventilation signal (Figure 2). Two main parameters were derived from the FFT: the frequency in hertz (or period in seconds) of the larger peak and its power estimated as the area under the peak at $\pm 0.02 \mathrm{~Hz}$ around the peak (in $\mathrm{L}^{2} \cdot \mathrm{min}^{-2}, \%^{2}$ and $\mathrm{mmHg}^{2}$, respectively for $\varsigma \mathrm{E}, \mathrm{SpO}_{2}$ and $\mathrm{PETCO}_{2}$ spectra). This method allowed us to precisely quantify the presence of oscillations in the signals that are not observable in the standard protocol routinely used in the hypoxic exercise test where the signals are averaged every 20 seconds (39).

\section{Prospective study}

In order to determine the main factors likely to influence ventilatory oscillations, we designed two separate protocols: one was centered on the effect of intensity of power output at exercise (group Intensity), the second on the effect of the level of hypoxia (group Altitude).

\section{Subjects.}

For both protocols, healthy and non-smoking male subjects volunteered and were given exhaustive information about the successive tests. All were in good physical condition, with a medium level of regular physical activities (from 2 to 10 hours per week). They showed no evidence of cardiovascular or pulmonary disease. Subjects' characteristics are presented in table 2.

\section{Procedure.}

All subjects were first asked to perform a standard ramp test protocol on a cycloergometer to determine their maximal aerobic power (MAP, Table 2): after a 3 minute warm-up at 60 watts, power output was increased by 30 -watt steps every two minutes until exhaustion.

Group Intensity: six tests were randomly performed with a minimum of 2-day interval. After one minute rest for material habituation and cardiorespiratory parameters stabilization, subjects were first asked to keep a resting sitting position on the ergometer for $6 \mathrm{~min}$, and then to pedal for $6 \mathrm{~min}$ at around $65 \mathrm{rpm}$ pedaling cadence at an exercise intensity of 20, 30 and $40 \%$ of MAP. The whole test was executed either in normoxia or in normobaric hypoxic condition simulating an altitude of $4,000 \mathrm{~m}$. 
Group Altitude: four tests were randomly conducted, at different simulated altitudes: sea level, 2,000m, 3,000m and 4,000m, with fractions of inspired oxygen of respectively 0.209, $0.168,0.145$ and 0.127 . A 6-min rest / 6-min exercise test was performed and exercise intensity was set at $30 \%$ of MAP.

The ventilatory response to $\mathrm{CO}_{2}$ (HCVR) was determined using a modified Read's rebreathing method (33). After breathing room air through a mouthpiece device to establish a baseline, the valve was switched to a 10-liter rebreathing bag containing a mixture of $93 \% \mathrm{O}_{2}$ and $7 \% \mathrm{CO}_{2}$. After two deep breaths in order to speed up the gas mixing in the lungs, the subject was asked to spontaneously breath in the bag until ventilation reached 60 L.min ${ }^{-1}$. Collected data was then compiled in a $\varsigma_{\mathrm{E}-\mathrm{PETCO}}$ graph to calculate HCVR from the slope of the linear regression between $\varsigma \mathrm{E}$ and $\mathrm{PETCO}_{2}$.

The same data acquisition and analysis as in the retrospective study was performed in the prospective study. In addition, instantaneous cardiac output was measured using a transthoracic impedance technique (Physioflow ${ }^{\circledR}$, Manatec Biomedical, France) throughout the different phases.

The protocols were approved by the Ile-de-France Ethics Committee (CPP-IDF2) and an individual written informed consent has been collected from all subjects.

Statistical analysis. Results are presented as mean \pm standard deviation. In the retrospective study, a two-way analysis of variance was performed to evaluate the difference in period and peak power of $\varsigma_{\varsigma} \mathrm{E}, \mathrm{SpO}_{2}$ and $\mathrm{PETCO}_{2}$ spectra between groups (low and high HVRe) and between conditions (rest, exercise, hypoxia, normoxia). A Bonferroni post-hoc test was then used when applicable. In the prospective study, regarding the expected difference in mean period (3 seconds), the standard deviation (2.4 seconds), a level of significance at $0.05 \%$ and a statistical power at $80 \%$, the minimum number of subjects to be included was 7 for each protocol. Since spectra peak power showed a high standard deviation and a non-normal distribution, we performed a logarithmic transformation of raw data, and calculated a minimum number of subjects to include of 10. A two-way analysis of variance was then performed to evaluate the difference in period and power of the $\varsigma \mathrm{E}, \mathrm{SpO}_{2}$ and $\mathrm{PETCO}_{2}$ spectra between conditions (rest and exercise, hypoxia and normoxia). A multivariate regression was done to establish potential correlations between period and peak power of $\varsigma \mathrm{E}, \mathrm{SpO}_{2}$ and $\mathrm{PETCO}_{2}$ spectra on one hand, and cardiorespiratory parameters on the other hand: $\varsigma \mathrm{E}, \mathrm{SpO}_{2}, \mathrm{PETCO}_{2}$, Ttot, Ti, VT, heart rate (HR) and cardiac output $(\Theta c)$. 


\section{Results}

\section{Retrospective study}

Typical recordings of a hypoxic exercise test with breath-by-breath output are shown in Figure 1. In the subject with a high HVRe (lower panel), oscillations of ${ }_{\varsigma} \mathrm{E}$, especially in the hypoxic exercise phase, are clearly shown. Note that the oscillations are also observable on the $\mathrm{SpO}_{2}$ and $\mathrm{PETCO}_{2}$ signals. Oscillations are less apparent in the subject with low HVRe (upper panel).

The corresponding frequency spectrum of the ventilation signal during the four phases of the hypoxic exercise test in a given subject (high HVRe) is presented in figure 2 as an example. Note that a peak was observable in all phases but particularly sharp in exercise in hypoxia (EH). Mean values of period of oscillation of ${ }_{\varsigma} \mathrm{E}$ and $\mathrm{SpO}_{2}$ signals, as well as power of the corresponding peak in the four phases of the test are presented in figure 3. Two-way ANOVA showed that $\varsigma_{\mathrm{E}}$, $\mathrm{SpO}_{2}$ and $\mathrm{PETCO}_{2}$ periods were lower at exercise $(\mathrm{P}<0.001)$ and similar in both groups. $\varsigma \mathrm{E}$ and $\mathrm{SpO}_{2}$ peak powers were higher at exercise $(\mathrm{P}<0.001)$ and higher in the high HVRe group $(\mathrm{P}<0.001)$. Detailed differences are showed in figure 3 for ${ }_{\varsigma} \mathrm{E}$ and $\mathrm{SpO}_{2}\left(\mathrm{PETCO}_{2}\right.$ not shown $)$. For the whole population, the average period was around $12.5 \mathrm{~s}$ at rest and significantly decreased to $11.1 \mathrm{~s}$ at exercise $(\mathrm{P}<0.001)$, without difference between the two HVRe groups. The mean period of the $\varsigma \mathrm{E}$ signal $(11.1 \pm 1.8 \mathrm{~s})$ was slightly shorter than the period for $\mathrm{SpO}_{2}(12.0 \pm 1.8$ s, $P<0.001)$ and $\mathrm{PETCO}_{2}(11.5 \pm 1.8 \mathrm{~s}, P<0.001)$. The differences are small but highly significant (paired comparison).

\section{Prospective study}

The same breath-by-breath recording methods were used in the prospective study. Varying altitude and exercise intensities allowed identifying some of the factors influencing period and peak power. Following data are presented by combining the results of the Intensity and Altitude groups.

As expected, $\varsigma_{5}$ increased with exercise and from normoxia to hypoxia, $\mathrm{SpO}_{2}$ decreased with hypoxia and with exercise (in hypoxia) and $\mathrm{PETCO}_{2}$ decreased with hypoxia and increased with exercise (Figure 4, left column).

As in the retrospective study, a peak in ${ }_{6} \mathrm{E}, \mathrm{SpO}_{2}$ and $\mathrm{PETCO}_{2}$ spectra was clearly noticeable, especially during exercise in the hypoxic phase. Mean values for period and peak power are presented in figure 4 . The period of oscillations during exercise was shorter than in rest, for all altitudes $(P<0.001)$, decreased with increasing exercise intensity $(P<0.001)$ and was not influenced by altitude. Peak power of $\varsigma \mathrm{E}$ oscillations increased with altitude $(P<0.001)$ and from 
rest to exercise $(P<0.001)$ but was not influenced by the level of exercise intensity. A similar trend was shown in the $\mathrm{SpO}_{2}$ and $\mathrm{PETCO}_{2}$ signals, although the variations were less marked (Figure 4).

Multivariate regressions evidenced significant correlations during exercise between $\varsigma \mathrm{E}$ period and $\varsigma \mathrm{E}(P<0.001)$ or $\Theta \mathrm{c}(P<0.001)$ and hypoxia $(P<0.01)$, as well as between $\varsigma \mathrm{E}$ peak power and $\varsigma \mathrm{E}(P<0.001)$ or $\Theta \mathrm{c}(P<0.001)$ and hypoxia $(P<0.001)$. Regressions for $\varsigma \mathrm{E}$ period and $\varsigma \mathrm{E}$ peak power, for each condition (normoxia and hypoxia) are shown in figure 5. $\varsigma \mathrm{E}$ period was negatively related to the intensity of $\varsigma_{\varsigma} \mathrm{E}$ and $\Theta c$ in hypoxic conditions. $\varsigma_{\mathrm{E}} \mathrm{E}$ peak power was positively related to $\varsigma \mathrm{E}$ and $\Theta c$ both in normoxic and hypoxic conditions (Figure 5). For a given level of $\varsigma \mathrm{E}$ (or $\Theta \mathrm{c}$ ), $\varsigma \mathrm{E}$ peak power and period were greater in hypoxia than in normoxia $(P<0.001)$. Similar correlations were found for the $\mathrm{PETCO}_{2}$ peak power with $\varsigma \mathrm{E}$ and $\Theta c$ (results not shown). No significant correlation was found for the $\mathrm{SpO}_{2}$ signal.

Interestingly, relating $\varsigma \mathrm{E}$ period with Ttot, we found a very significant correlation both at rest and exercise (Figure 6). Two-factor regression analysis of $\varsigma$ E period as a function of Ttot and exercise showed a significant correlation with Ttot $(P<0.001)$ and with exercise $(P<0.05)$.

When pooling the individual values of oscillation periods at exercise in hypoxic conditions, the mean period of the $\varsigma \mathrm{E}$ signal was $10.9 \pm 1.3 \mathrm{~s}$, slightly shorter than the $\mathrm{SpO}_{2}$ period $(11.6 \pm 1.5 \mathrm{~s}$, $P<0.001)$ and $\mathrm{PETCO}_{2}$ period $(11.6 \pm 1.3 \mathrm{~s}, P<0.001)$, similarly to what was found in the retrospective study.

Relating $\varsigma$ E peak power to HCVR, we found a significant correlation in hypoxic $(3,000 \mathrm{~m})$ conditions but not in normoxia (Figure 7, left). Thus the intensity of $\varsigma^{E}$ oscillations is higher in subjects with a higher ventilatory response to $\mathrm{CO}_{2}$, but only in hypoxic conditions. A similar relationship was found for $\mathrm{PETCO}_{2}$ peak power (Figure 7, right) but not for $\mathrm{SpO}_{2}$ (results not shown). No correlation was found between $\varsigma \mathrm{E}, \mathrm{SpO}_{2}$ or $\mathrm{PETCO}_{2}$ period and HCVR (results not shown).

\section{Discussion}

To our knowledge, this is the first description of a spontaneous oscillation of ventilation in awake normal subjects at rest and during exercise in normoxic and hypoxic conditions. We observed a period of oscillations between 8 and 16 seconds, meaning that spontaneous ventilation oscillates between a maximum and a minimum every 3 to 5 breaths at rest and 4 to 7 
breaths during exercise. No such observation has been made in previous studies of ventilation in hypoxic exercise conditions, probably because it requires a breath-by-breath recording of the ventilation signal and in most cases a spectral analysis to evidence the periodicity of the signal.

Both retrospective and prospective study show that the period is shorter at exercise when cardiac output and ventilation are higher. In addition, the oscillation is more pronounced in subjects with a higher sensitivity to hypoxia of their peripheral chemoreceptors. These findings provide novel evidence that support the theory of the closed loop system of control of ventilation, involving the sensitivity of the central and peripheral chemoreceptors and the time delay of the $\mathrm{O}_{2} / \mathrm{CO}_{2}$ signals between the receptors and the respiratory control centers $(19,21$, 29). The lung to carotid body circulation time may also affect the oscillation period (43). Exercise may shorten the delay through the increased blood velocity and therefore decrease the period of oscillation. The high gain of the feedback control system in high responders to hypoxia may increase the instability of the system and favor the appearance of oscillations. This spontaneous periodic breathing was probably uncovered by the brisk stimulation of the closed loop control system by imposing a hypoxic exercise to the subject, probably inducing rapid variations in the $\mathrm{O}_{2}$ and $\mathrm{CO}_{2}$ signals.

The combination of exercise and hypoxia may trigger the instability of the system since exercise by itself induces a further drop in $\mathrm{O}_{2}$ arterial pressure and saturation in hypoxic conditions (44). This is confirmed in the present study by the fact that, at the same level of ventilation during exercise, oscillations of ventilation are much greater in hypoxia than in normoxia (Figure 5, lower left panel), supporting the hypothesis that hypoxia, through peripheral chemoreceptors, may be a main source of the system instability. This periodic breathing is not readily observable at rest in normoxia in awake subjects, probably because of the chaotic pattern of spontaneous ventilation and the influence of the cortical control (45).

Our results indicate that $\varsigma \mathrm{E}$ peak power rises with ventilatory response to $\mathrm{CO}_{2}$ in hypoxia. This suggests that the well-established synergistic effect of hypoxia and hypercapnia on the control of ventilation $(15,32)$ is involved in the genesis of ventilatory oscillations.

Oscillations were found in the ventilation signal as well as in $\mathrm{SpO}_{2}$ and $\mathrm{PETCO}_{2}$, which are indirect markers of the two main physiological variables controlling ventilation, i.e. arterial $\mathrm{PO}_{2}$ and $\mathrm{PCO}_{2}$. The fact that these three signals were obtained through completely independent techniques reinforces the validity of the observed periodic pattern. The slight but highly significant difference in the period for $\varsigma \mathrm{E}, \mathrm{SpO}_{2}$ and $\mathrm{PETCO}_{2}$ is surprising, but could be in fact explained by either a non-linearity of the control system involving $\mathrm{O}_{2}$ and $\mathrm{CO}_{2}$ signals, or the 
existence of the lung $\mathrm{O}_{2} / \mathrm{CO}_{2}$ stores which would act as a low pass filter reducing the oscillation frequency of the $\mathrm{SpO}_{2}$ and $\mathrm{PETCO}_{2}$ signals.

The mean period of $\varsigma_{\mathrm{E}} \mathrm{E}$ oscillation $(11 \mathrm{~s})$ observed at exercise in the present study is shorter than the one observed at rest at high altitude $(20 \mathrm{~s}$ at $3050 \mathrm{~m})(8)$, during sleep at high altitude $(15 \mathrm{~s}$ at $8000 \mathrm{~m})(19)$ or in patients suffering from cardiac failure (around 1 minute) $(12,36,37)$ or obstructive sleep apneas (> $38 \mathrm{~s})(12,40)$. It stands in the large range of oscillations (5 to $100 \mathrm{~s})$ observed in uncontrolled conditions in subjects climbing at high altitude (20). It is clearly out of the range of the periods observed in the present hypoxia exercise test for breathing frequency ( 3 to $5 \mathrm{~s})$, heart rate $(0.4$ to $1.0 \mathrm{~s})$ or pedaling rate $(1 \mathrm{~s})$. It is interesting to note that, in the present study, an increase in cardiac output (as expected during exercise) exacerbated the oscillations whereas in patients with cardiac failure, the predominant hypothesis to explain the CheyneStokes breathing pattern is an increase in time delay due to low cardiac output $(11,18)$. A phase shift between the various physiological signals involved in the control loop might contribute to the instability of the system, as seen with heart rate and blood pressure, with arterial $\mathrm{O}_{2}$ saturation, or tidal volume in normal subjects during sleep in hypoxia $(3,29)$ or in patients with cardiac failure $(4,36)$. The observation that exercise appears to increase ventilation oscillations suggests that whatever effect enhances oscillations is strong enough to offset the stabilizing effect of the reduced phase shift. In obstructive sleep apneas, an initial apnea may trigger oscillations in an already unstable system, and airway obstructions (permanent or intermittent) may promote hypoxia and make the system more unstable.

In conclusion, a spontaneous periodic breathing pattern with a period of around 11 seconds at exercise has been evidenced in normal subjects, uncovering the intrinsic unstable structure of the ventilation control loop. The intensity of these oscillations depends on the ventilatory response to hypoxia, suggesting that peripheral chemoreceptors play a role in the genesis or modulation of this phenomenon. Further studies will be necessary to determine the respective influence of $\mathrm{O}_{2}$ and $\mathrm{CO}_{2}$ signals, sensitivity of the central and peripheral chemoreceptors, and intensity of cardiac output on the period and power of this observed phenomenon. This observation may have an impact on the comprehension of central or obstructive apneas and periodic breathing in diseases and on early detection of high-risk subjects with a hypoxic exercise test. 


\section{References}

1. Ainslie PN, Lucas SJE, Burgess KR. Breathing and sleep at high altitude. Respir Physiol Neurobiol 188: 233-256, 2013.

2. Bålfors EM, Franklin KA. Impairment of cerebral perfusion during obstructive sleep apneas. American Journal of Respiratory and Critical Care Medicine 150: 1587-1591, 1994.

3. Berssenbrugge A, Dempsey J, Iber C, Skatrud J, Wilson P. Mechanisms of hypoxia-induced periodic breathing during sleep in humans. J Physiol (Lond) 343: 507-524, 1983.

4. Binggeli C, Sudano I, Corti R, Spieker L, Jenni R, Lüscher TF, Noll G. Spontanous periodic breathing is associated with sympathetic hyperreactivity and baroreceptor dysfunction in hypertension. J Hypertens 28: 985-992, 2010.

5. Blain GM, Smith CA, Henderson KS, Dempsey JA. Peripheral chemoreceptors determine the respiratory sensitivity of central chemoreceptors to $\mathrm{CO} 2$ : Peripheral-central chemoreceptor interdependence. The Journal of Physiology 588: 2455-2471, 2010.

6. Bourdillon N, Fan J-L, Kayser B. Cerebral oxygenation during the Richalet hypoxia sensitivity test and cycling time-trial performance in severe hypoxia. Eur J Appl Physiol 114: 1037-1048, 2014.

7. Brack T, Randerath W, Bloch KE. Cheyne-Stokes Respiration in Patients with Heart Failure: Prevalence, Causes, Consequences and Treatments. Respiration 83: 165-176, 2012.

8. Brusil PJ, Waggener TB, Kronauer RE, Gulesian P Jr. Methods for identifying respiratory oscillations disclose altitude effects. J Appl Physiol Respir Environ Exerc Physiol 48: 545-556, 1980.

9. Canouï-Poitrine F, Veerabudun K, Larmignat P, Letournel M, Bastuji-Garin S, Richalet J-P. Risk prediction score for severe high altitude illness: a cohort study. PLoS ONE 9: e100642, 2014.

10. Chadha TS, Birch S, Sackner MA. Periodic breathing triggered by hypoxia in normal awake adults. Modification by naloxone. Chest 88: 16-23, 1985.

11. Cherniack NS. Mathematical models of periodic breathing and their usefulness in understanding cardiovascular and respiratory disorders. Experimental Physiology 91: 295-305, 2005.

12. Dempsey JA, Veasey SC, Morgan BJ, O’Donnell CP. Pathophysiology of sleep apnea. Physiol Rev 90: 47-112, 2010.

13. Dhakal BP, Murphy RM, Lewis GD. Exercise oscillatory ventilation in heart failure. Trends Cardiovasc Med 22: 185-191, 2012.

14. Ben-Dov I, Sietsema KE, Casaburi R, Wasserman K. Evidence that circulatory oscillations accompany ventilatory oscillations during exercise in patients with heart failure. Am Rev Respir Dis 145: 776-781, 1992.

15. Duffin J. Measuring the respiratory chemoreflexes in humans. Respir Physiol Neurobiol 177: 7179, 2011.

16. Duffin J, Mateika JH. Cross-Talk opposing view: peripheral and central chemoreflexes have additive effects on ventilation in humans. J Physiol (Lond) 591: 4351-4353, 2013. 
17. Fan J-L, Burgess KR, Thomas KN, Lucas SJE, Cotter JD, Kayser B, Peebles KC, Ainslie PN. Effects of acetazolamide on cerebrovascular function and breathing stability at 5,050 m. The Journal of Physiology ( January 2012). doi: 10.1113/jphysiol.2011.219923.

18. Fowler AC, Kalamangalam GP. The role of the central chemoreceptor in causing periodic breathing. IMA J Math Appl Med Biol 17: 147-167, 2000.

19. Fowler AC, Kalamangalam GP. Periodic breathing at high altitude. IMA J Math Appl Med Biol 19: 293-313, 2002.

20. Garde A, Giraldo BF, Jane R, Latshang TD, Turk AJ, Hess T, Bosch MM, Barthelmes D, Hefti JP, Maggiorini M, Hefti U, Merz TM, Schoch OD, Bloch KE. Periodic breathing during ascent to extreme altitude quantified by spectral analysis of the respiratory volume signal. Conf Proc IEEE Eng Med Biol Soc 2012: 707-710, 2012.

21. Jubran A, Tobin MJ. Effect of isocapnic hypoxia on variational activity of breathing. Am J Respir Crit Care Med 162: 1202-1209, 2000.

22. Khoo MC. Determinants of ventilatory instability and variability. Respir Physiol 122: 167-182, 2000.

23. Kumar P, Prabhakar NR. Peripheral Chemoreceptors: Function and Plasticity of the Carotid Body [Online]. In: Comprehensive Physiology, edited by Terjung R. John Wiley \& Sons, Inc. http://doi.wiley.com/10.1002/cphy.c100069 [31 Mar. 2014].

24. Küpper T, Schöffl V, Netzer N. Cheyne stokes breathing at high altitude: a helpful response or a troublemaker? Sleep Breath 12: 123-127, 2008.

25. Lahiri S, Forster RE 2nd. $\mathrm{CO} 2 / \mathrm{H}(+)$ sensing: peripheral and central chemoreception. Int J Biochem Cell Biol 35: 1413-1435, 2003.

26. Lahiri S, Roy A, Baby SM, Hoshi T, Semenza GL, Prabhakar NR. Oxygen sensing in the body. Prog Biophys Mol Biol 91: 249-286, 2006.

27. Lhuissier FJ, Brumm M, Ramier D, Richalet J-P. Ventilatory and cardiac responses to hypoxia at submaximal exercise are independent of altitude and exercise intensity. J Appl Physiol 112: 566$570,2012$.

28. Lhuissier FJ, Canouï-Poitrine F, Richalet J-P. Ageing and cardiorespiratory response to hypoxia. J Physiol (Lond) 590: 5461-5474, 2012.

29. Lipsitz LA, Hashimoto F, Lubowsky LP, Mietus J, Moody GB, Appenzeller O, Goldberger AL. Heart rate and respiratory rhythm dynamics on ascent to high altitude. Br Heart J 74: 390-396, 1995.

30. Masuyama S, Kohchiyama S, Shinozaki T, Okita S, Kunitomo F, Tojima H, Kimura H, Kuriyama T, Honda Y. Periodic breathing at high altitude and ventilatory responses to $\mathrm{O} 2$ and CO2. Jpn J Physiol 39: 523-535, 1989.

31. Mateika JH, Mendello C, Obeid D, Badr MS. Peripheral chemoreflex responsiveness is increased at elevated levels of carbon dioxide after episodic hypoxia in awake humans. J Appl Physiol 96: 1197-1205; discussion 1196, 2004.

32. Mohan R, Duffin J. The effect of hypoxia on the ventilatory response to carbon dioxide in man. Respir Physiol 108: 101-115, 1997. 
33. Mohan RM, Amara CE, Cunningham DA, Duffin J. Measuring central-chemoreflex sensitivity in man: rebreathing and steady-state methods compared. Respir Physiol 115: 23-33, 1999.

34. Pack AI, Gottschalk A. Mechanisms of ventilatory periodicities. Ann Biomed Eng 21: 537-544, 1993.

35. Pinna GD, Maestri R, Mortara A, La Rovere MT. Cardiorespiratory interactions during periodic breathing in awake chronic heart failure patients. Am J Physiol Heart Circ Physiol 278: H932-941, 2000 .

36. Pinna GD, Maestri R, Mortara A, La Rovere MT, Fanfulla F, Sleight P. Periodic breathing in heart failure patients: testing the hypothesis of instability of the chemoreflex loop. J Appl Physiol 89: 2147-2157, 2000.

37. Ponikowski P, Anker SD, Chua TP, Francis D, Banasiak W, Poole-Wilson PA, Coats AJ, Piepoli M. Oscillatory breathing patterns during wakefulness in patients with chronic heart failure: clinical implications and role of augmented peripheral chemosensitivity. Circulation 100: 24182424, 1999.

38. Read DJ. A clinical method for assessing the ventilatory response to carbon dioxide. Australas Ann Med 16: 20-32, 1967.

39. Richalet J-P, Larmignat P, Poitrine E, Letournel M, Canouï-Poitrine F. Physiological risk factors for severe high-altitude illness: a prospective cohort study. Am J Respir Crit Care Med 185: 192-198, 2012.

40. Ryan CM, Bradley TD. Periodicity of obstructive sleep apnea in patients with and without heart failure. Chest 127: 536-542, 2005.

41. Schulz R, Blau A, Börgel J, Duchna HW, Fietze I, Koper I, Prenzel R, Schädlich S, Schmitt J, Tasci S, Andreas S, working group Kreislauf und Schlaf of the German Sleep Society (DGSM). Sleep apnoea in heart failure. Eur Respir J 29: 1201-1205, 2007.

42. Waggener TB, Brusil PJ, Kronauer RE, Gabel RA, Inbar GF. Strength and cycle time of highaltitude ventilatory patterns in unacclimatized humans. J Appl Physiol 56: 576-581, 1984.

43. Ward SA, Drysdale DB, Cunningham DJ, Petersen ES. Inspiratory-expiratory responses to alternate-breath oscillation of PACO2 and PAO2. Respir Physiol 36: 311-325, 1979.

44. Woorons X, Mollard P, Pichon A, Lamberto C, Duvallet A, Richalet J-P. Moderate exercise in hypoxia induces a greater arterial desaturation in trained than untrained men. Scand J Med Sci Sports 17: 431-436, 2007.

45. Wysocki M, Fiamma M-N, Straus C, Sang Poon C, Similowski T. Chaotic dynamics of ventilatory flow in humans. Conf Proc IEEE Eng Med Biol Soc 1: 759-762, 2005.

46. Xie A, Skatrud JB, Puleo DS, Dempsey JA. Influence of arterial O2 on the susceptibility to posthyperventilation apnea during sleep. J Appl Physiol 100: 171-177, 2006. 


\section{Figure captions.}

Figure 1. Breath-by-breath output of minute ventilation $(\varsigma \mathrm{E})$, pulse $\mathrm{O}_{2}$ saturation $\left(\mathrm{SpO}_{2}\right)$ and end tidal $\mathrm{PCO}_{2}\left(\mathrm{PETCO}_{2}\right)$ during a hypoxia exercise test. Lower panel: subject with a high HVRe (0.93 L. $\left.\mathrm{min}^{-1} \cdot \mathrm{kg}^{-1}\right)$. Upper panel: subject with a low HVRe $\left(0.60 \mathrm{~L} \cdot \mathrm{min}^{-1} \cdot \mathrm{kg}^{-1}\right)$.

Figure 2. Example of spectral analysis of minute ventilation by Fast Fourier Transform in the four phases of a hypoxia exercise test. RN: rest in normoxia, $\mathrm{RH}$ : rest in hypoxia, EH: exercise in hypoxia, EN: exercise in normoxia. PSD: Power Spectral Density. Note that the frequency of the main peak in hypoxic exercise conditions $(\mathrm{EH})$ is around $0.08 \mathrm{~Hz}$, which corresponds to a period of 12.5 seconds.

Figure 3. $\varsigma \mathrm{E}$ and $\mathrm{SpO}_{2}$ period and power of the main peak obtained from the spectral analysis of minute ventilation during the various phases of a hypoxia exercise test. RN: rest in normoxia, $\mathrm{RH}$ : rest in hypoxia, EH: exercise in hypoxia, EN: exercise in normoxia. Mean $\pm \mathrm{SD}$. Condition vs RN: *, $\mathrm{P}<0.05 ; * *, \mathrm{P}<0.01 ; * * *, \mathrm{P}<0.001$. High HVRe vs Low HVRe: +, $\mathrm{P}<0.05 ;++$, $\mathrm{P}<0.01$.

Figure 4. Mean values of $\varsigma \mathrm{E}, \mathrm{SpO}_{2}$ and $\mathrm{PETCO}_{2}$ and corresponding periods and peak powers extracted from the prospective study. Mean \pm SD. Condition vs Rest:,$+ \mathrm{P}<0.05 ;++, \mathrm{P}<0.01$; ,$+++ \mathrm{P}<0.001$. Condition vs Sea level: *, $\mathrm{P}<0.05 ; * *, \mathrm{P}<0.01 ; * * *, \mathrm{P}<0.001$.

Figure 5. Linear regressions between $\varsigma \mathrm{E}$ period and peak power, and $\varsigma \mathrm{E}$ (left column) and Cardiac Output (right column) during exercise, in normoxic and hypoxic conditions, extracted from the prospective study.

Figure 6. Linear regressions between $\varsigma \mathrm{E}$ period and total respiratory cycle time (Ttot) at rest and exercise, extracted from the prospective study.

Figure 7. Linear regressions between $\varsigma \mathrm{E}$ (left) and $\mathrm{PETCO}_{2}$ (right) peak powers during exercise and hypercapnic ventilatory response (HCVR), extracted from the prospective study. 
Table 1. Characteristics of the subjects following their hypoxic ventilatory response at exercise (HVRe).

\begin{tabular}{|c|c|c|c|c|c|c|}
\hline & $\mathbf{n}$ & $\begin{array}{c}\text { Sex } \\
\text { (female) }\end{array}$ & $\begin{array}{l}\text { Age } \\
\text { (yrs) }\end{array}$ & $\begin{array}{c}\text { Body } \\
\text { weight }(\mathrm{kg})\end{array}$ & $\begin{array}{c}\text { Height } \\
(\mathrm{cm})\end{array}$ & $\begin{array}{c}\text { HVRe } \\
\left(\mathrm{L} \cdot \mathrm{min}^{-1} \cdot \mathrm{kg}^{-1}\right)\end{array}$ \\
\hline High HVRe & 41 & 18 & $47 \pm 14$ & $71 \pm 9$ & $171 \pm 7$ & $1.26 \pm 0.40$ \\
\hline Low HVRe & 41 & 20 & $49 \pm 16$ & $66 \pm 13$ & $167 \pm 9$ & $0.55 \pm 0.15$ \\
\hline$P$ High vs Low & & $\mathrm{ns}$ & $\mathrm{ns}$ & $\mathrm{ns}$ & ns & $<0.001$ \\
\hline
\end{tabular}

Mean \pm SD

Table 2. Characteristics of the subjects of the Intensity and Altitude studies.

\begin{tabular}{cccccc}
\hline & $\mathbf{n}$ & $\begin{array}{c}\text { Age } \\
(\text { years })\end{array}$ & $\begin{array}{c}\text { Height } \\
(\mathrm{cm})\end{array}$ & $\begin{array}{c}\text { Weight } \\
(\mathrm{kg})\end{array}$ & $\begin{array}{c}\text { Maximal aerobic power (MAP) } \\
\text { (Watts) }\end{array}$ \\
\hline $\begin{array}{c}\text { Group } \\
\text { Intensity } \\
\text { Group }\end{array}$ & 12 & $28.6 \pm 6.7$ & $175.1 \pm 7.4$ & $75.1 \pm 14.7$ & $270.0 \pm 67.0$ \\
Altitude & 13 & $27.2 \pm 6.5$ & $173.8 \pm 9.0$ & $69.4 \pm 12.4$ & $235.8 \pm 47.9$ \\
\hline
\end{tabular}

Mean \pm SD 

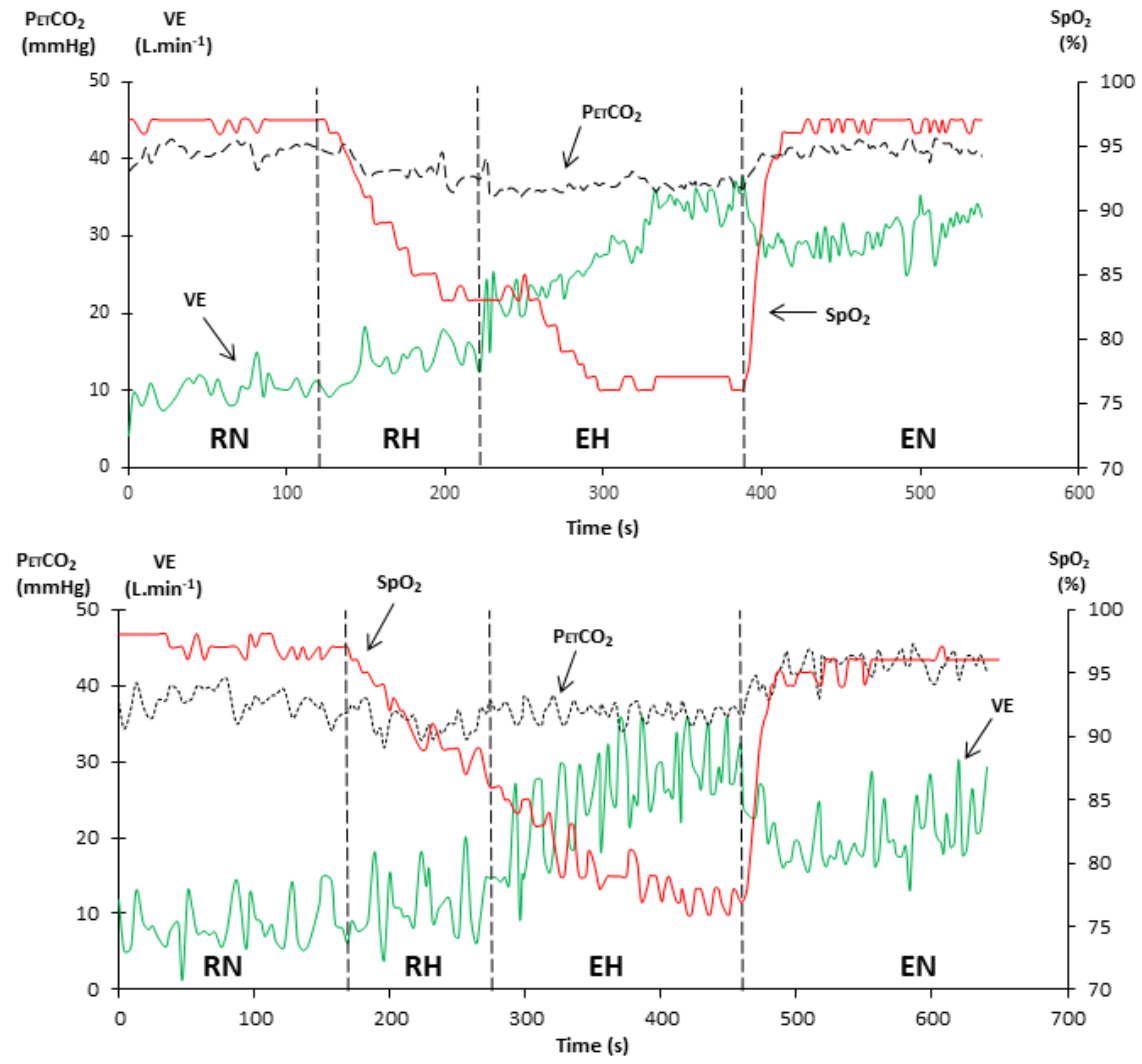

Figure 1.

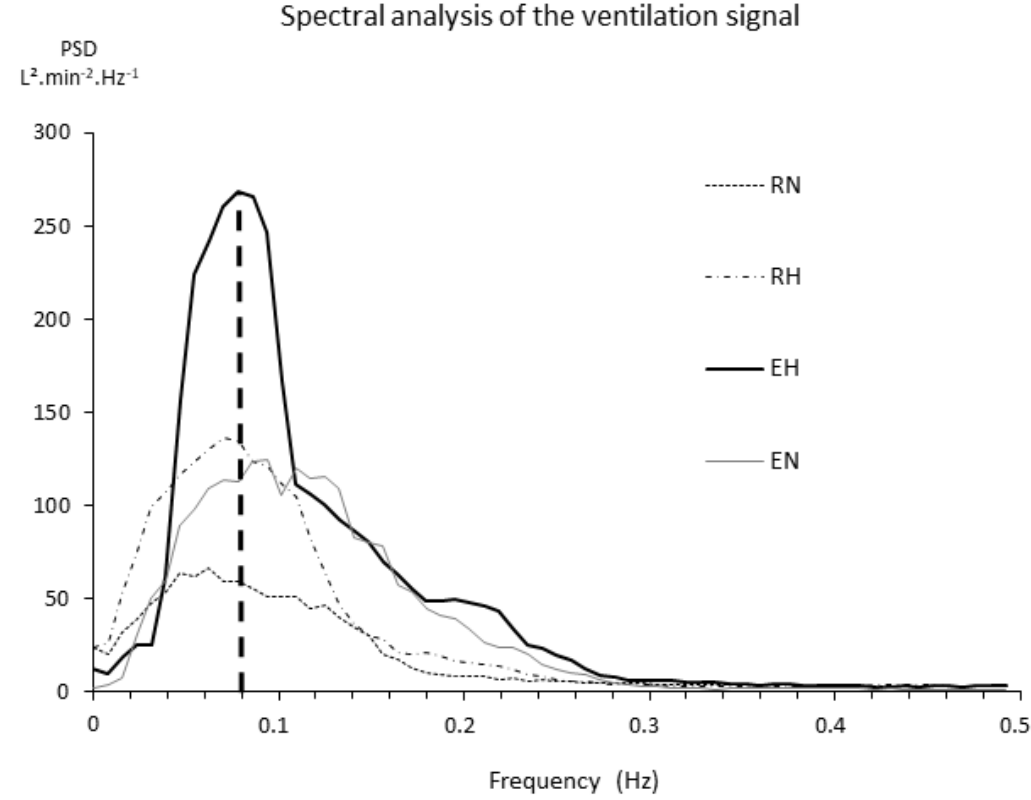

Figure 2 

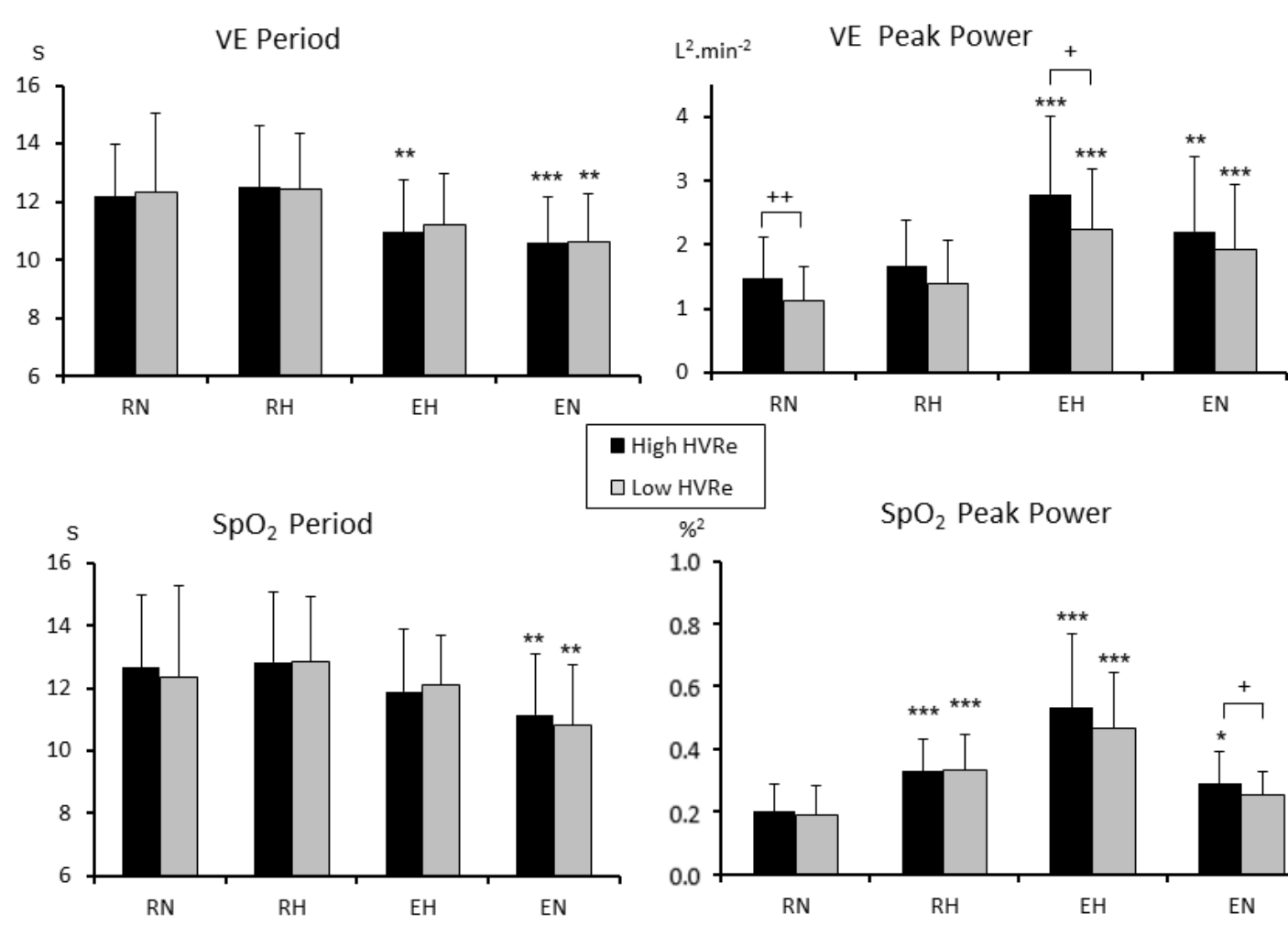

a High HVRe

$\square$ Low HVRe
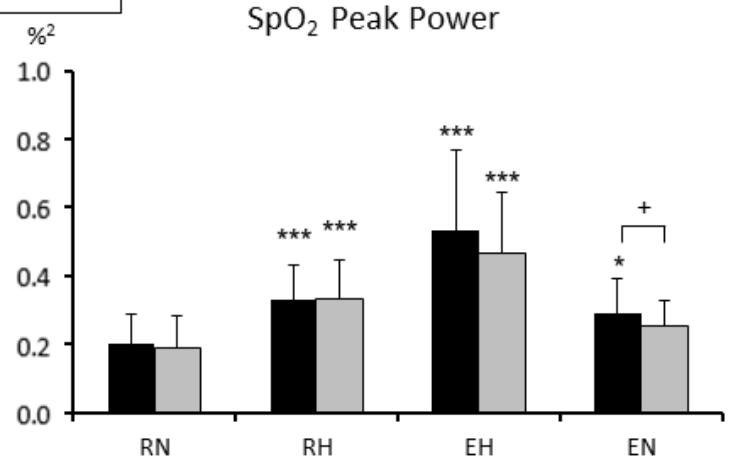

Figure 3
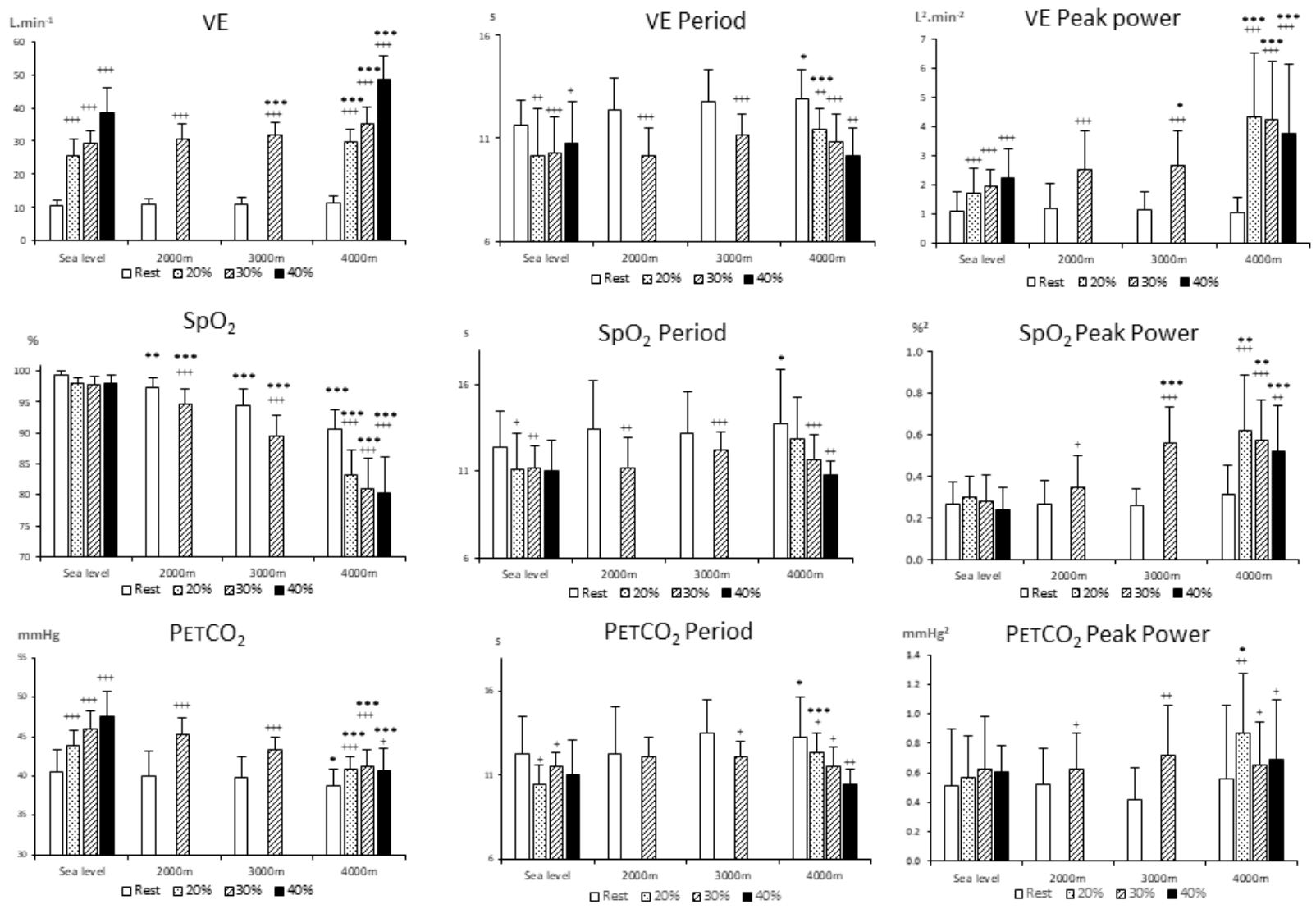

Figure 4 . 

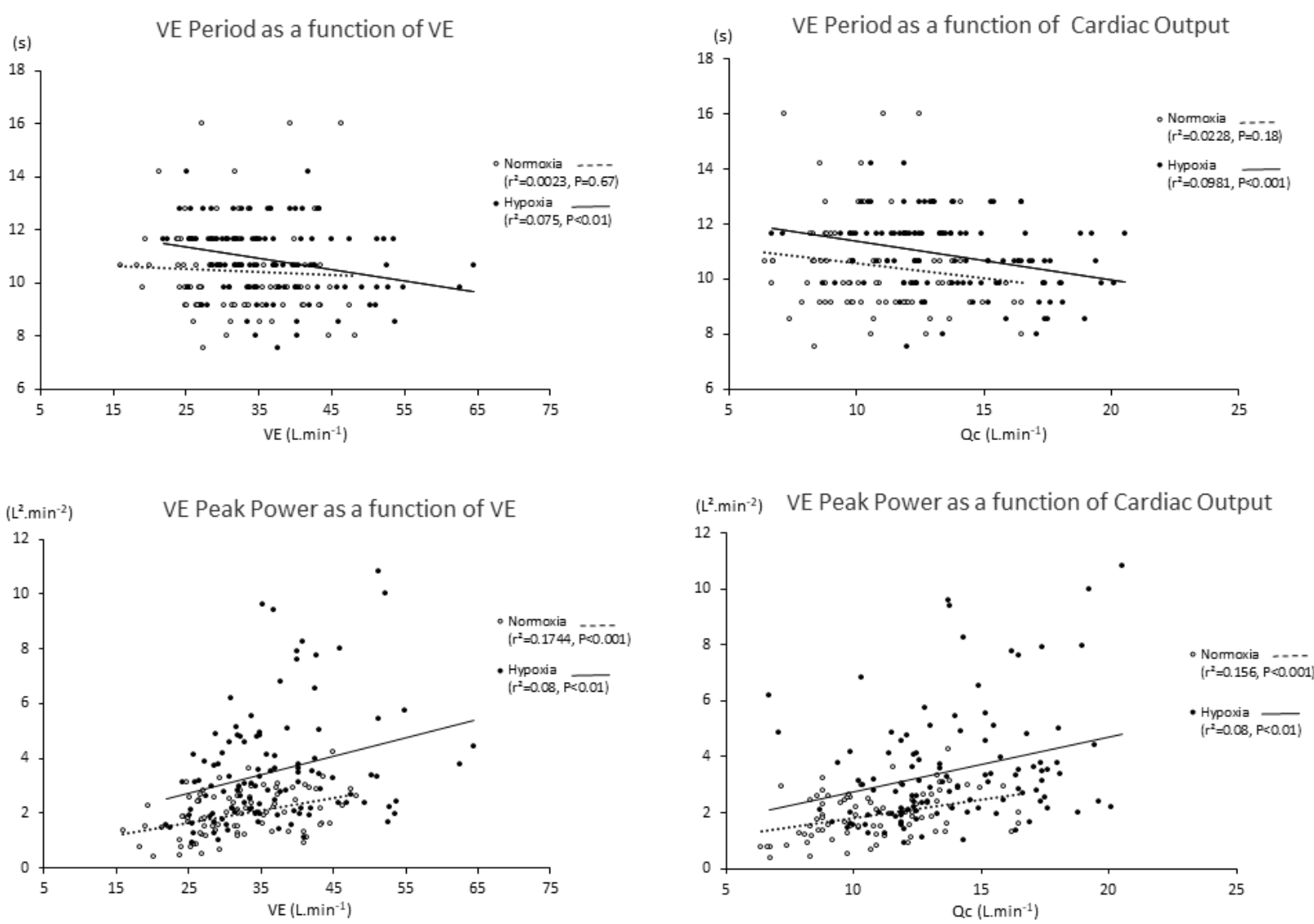

Figure 5

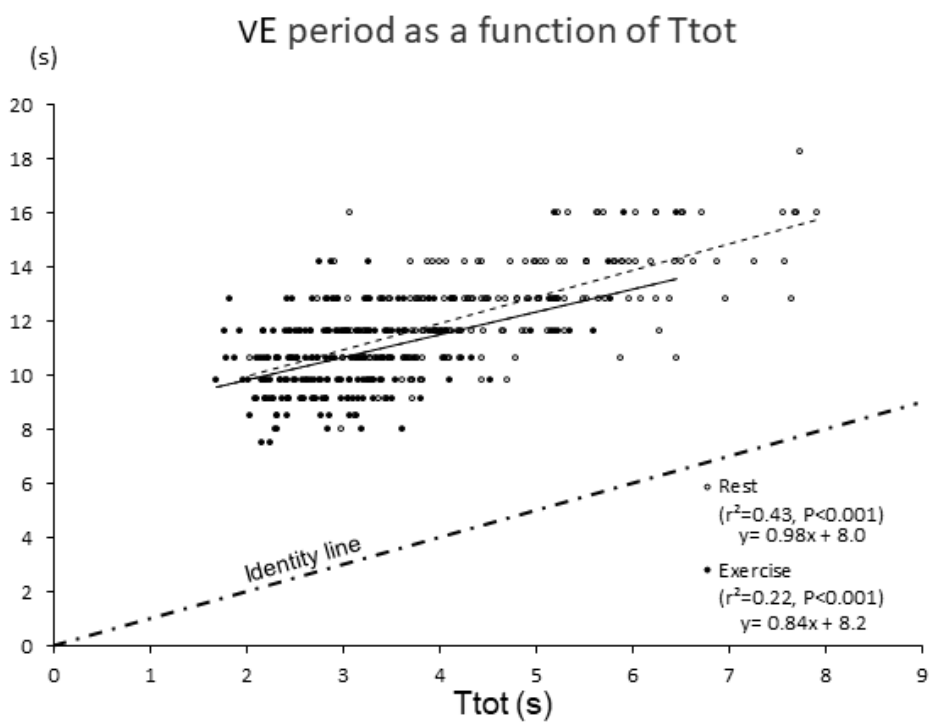

Figure 6. 

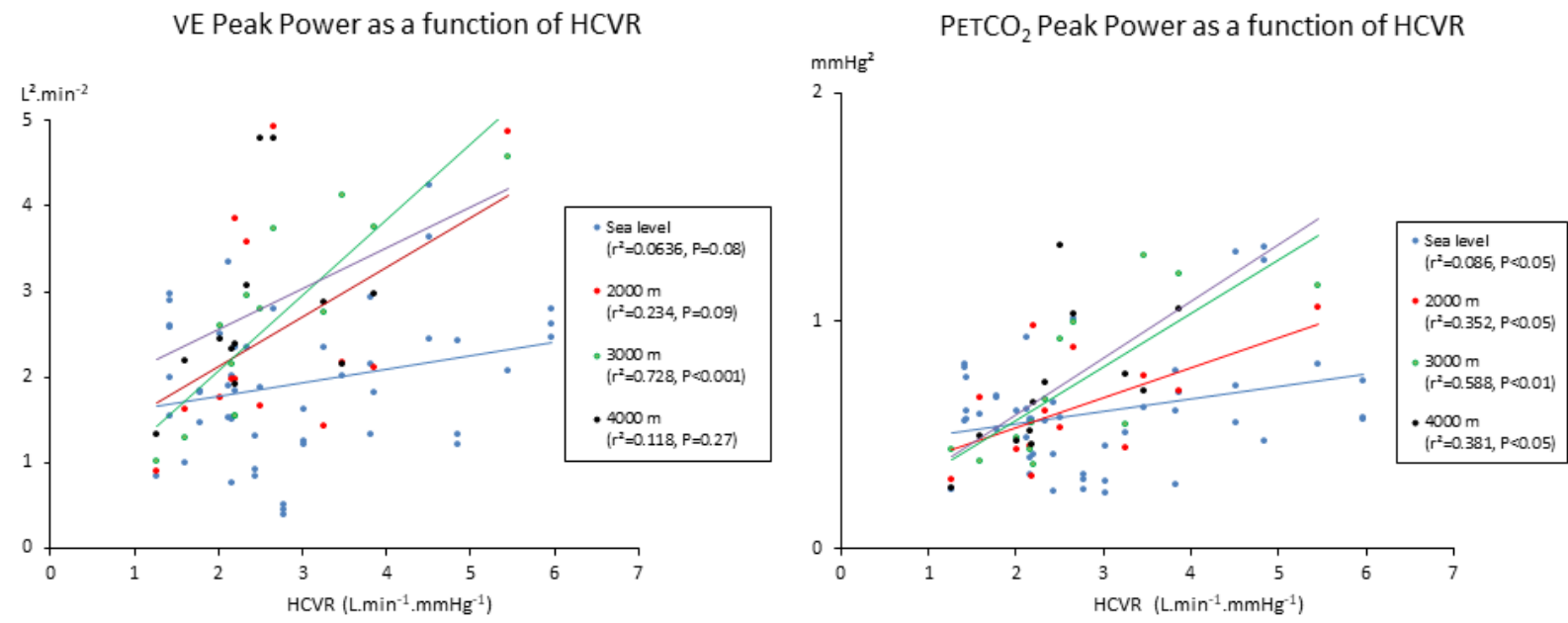

Figure 7. 